\title{
Pellet fuelling into radiative improved confinement discharges in TEXTOR-94
}

\author{
J. Hobirk ${ }^{\mathrm{a} *}$, A.M. Messiaen ${ }^{\mathrm{b}}$, K.H. Finken ${ }^{\mathrm{a}}$, J. Ongena ${ }^{\mathrm{b}}$, M. Brix ${ }^{\mathrm{a}}$, \\ R. Jaspers ${ }^{c}$, H.R. Koslowski ${ }^{\mathrm{a}}$, A. Krämer-Flecken ${ }^{\mathrm{a}}$, G. Mank ${ }^{\mathrm{a}}$, J. Rapp ${ }^{\mathrm{a}}$, \\ G. Telesca ${ }^{\mathrm{a}}$, B. Unterberg ${ }^{\mathrm{a}}$ \\ Trilateral Euregio Cluster: \\ a Institut für Plasmaphysik, Forschungszentrum Jülich GmbH, \\ Euratom Association, Jülich, Germany \\ b Laboratoire de Physique des Plasmas/Laboratorium voor Plasmafysica, \\ École Royale Militaire-Koninklijke Militaire School, \\ Association Euratom-Belgian State, Brussels, Belgium \\ ${ }^{c}$ FOM-Instituut voor Plasmafysica Rijnhuizen, Euratom Association, Nieuwegein, \\ Netherlands
}

\begin{abstract}
Normally pellet injection in strongly heated discharges leads at most to a relatively short improvement of the energy and particle confinement times. In contrast to this finding, the radiative improved (RI) mode plasma of TEXTOR-94 is a very well suited target for pellet injection: the interaction of a pellet with the high density plasma together with the radiatively cooled edge leads in the best cases to an improved energy confinement time which lasts to the end of the heating phase. The pellet injection causes at first a density increase; this phase is followed by a quick partial particle loss and later an increase to a quasi-stationary value. Because of the linear density dependence of RI mode confinement this leads to an additional increase in energy confinement time.
\end{abstract}

\section{Introduction}

Fuelling by pellet injection into a magnetic confinement device [1] is an alternative way to the presently used gas fuelling or fuelling by high energy neutral beams for the plasma core. In a fusion reactor, pellet fuelling is foreseen such that the pellets cross the scrape-off layer and penetrate into the boundary of the plasma. In this way the fuelling efficiency, in particular for tritium, can be enhanced. In present day devices, pellet fuelling is also applied in order to enhance confinement. Confinement enhancement has often been observed in high density ohmic discharges [2-4]. In this type of discharge the injected pellet cools the discharge transiently and at the same time an inwards drift leads to an increase in central density. The plasma energy increases later together with temperature and density and the linear ohmic confinement law is extended to densities where otherwise saturated ohmic confinement would be found [2]. The discharge conditions after pellet injection result in a good target for auxiliary heating, leading

\footnotetext{
* Present address: Max-Planck-Institut für Plasmaphysik, Garching, Germany.
}

to the pellet enhanced performance (PEP) mode observed in different devices [5-7]; the energy content can improve transiently for some hundreds of milliseconds up to $1 \mathrm{~s}$ at JET and is accompanied by an instantaneous density peaking caused by central mass deposition from the pellet and a hollow temperature profile. A transport analysis at JET yields an inverse $q$ profile which is sometimes attributed as the cause of the improved confinement. On other experiments (such as TFTR) magnetic shear was not necessarily reversed and the conditions could be reached with weak or low shear in the plasma core.

In strongly heated plasmas with low confinement ( $\mathrm{L}$ mode), the positive influence of the pellet is decreased with increasing heating power $[3,8]$. In discharges with high confinement (H mode), in many cases a decrease in confinement is found if the injection is performed from the low field side $[9,10]$ but by injecting pellets from the high field side [9] confinement degradation can be avoided and the discharges reach high densities with good confinement. Sometimes pellet injection has triggered an $\mathrm{H}$ mode $[11,12]$.

All these enhanced confinement scenarios are transient. The improved confinement holds only for a few hundred milliseconds after each pellet. 


\section{J. Hobirk et al.}

\section{Experimental conditions}

TEXTOR-94 is a medium size circular tokamak $(R=1.75 \mathrm{~m}, a=0.46 \mathrm{~m})$. It is equipped with two neutral beam injectors of $2 \mathrm{MW}$ heating power each and an ICRH system consisting of two pairs of antennas (2 MW for each pair). The neutral beams are injected tangentially, one in the co-direction and one in the counter-direction. Another characteristic feature of TEXTOR-94 is the pumped toroidal belt limiter ALT-II [13-15] by which the particle content of the plasma can be controlled. The pumped limiter together with a feedback controlled gas inlet system [16] create the ability to control the content of gaseous impurities introduced into the plasma. Electron density and current profile measurements are performed with a nine channel HCN interferometer/polarimeter [17]. Temperature profiles are determined with a second and third harmonic ECE system [18].

TEXTOR-94 is equipped with a gas gun type nine shot pellet injector which provides pellets with a diameter of $1.5 \mathrm{~mm}$ and a length between 1 and $4 \mathrm{~mm}$ $[19,20]$. The particle content in each pellet can be adjusted between $5 \times 10^{18}$ and $1 \times 10^{20}$, corresponding to up to $200 \%$ of the total number of electrons in the plasma before injection. The size of the pellet can be influenced by a gradient heating system and the deuterium pressure in the freezing cell. The pellets can be injected into a discharge with preprogrammed injection times. The velocity depends on the pellet size and the pressure of the acceleration gas and ranges from 700 to $1200 \mathrm{~m} / \mathrm{s}$.

\section{Conditions for radiative improved mode}

The radiative improved (RI) mode [21, 22] in TEXTOR-94 is obtained by seeding medium- $Z$ impurities into auxiliary heated discharges. The impurities radiate in a toroidal and poloidal symmetric zone near the plasma boundary. The radiated power becomes a significant fraction of the power launched into the plasma. Together with the development of a radiative mantle, the energy content in the discharge and also the central density increase. Under these conditions the plasma exhibits energy confinement between ELMy and ELM-free H modes. In contrast to $\mathrm{H}$ mode, $\mathrm{RI}$ mode has no edge barrier and the resulting detrimental effects such as ELMs are avoided. The profile shapes of RI mode and $\mathrm{H}$ mode are in general very different; $\mathrm{H}$ mode

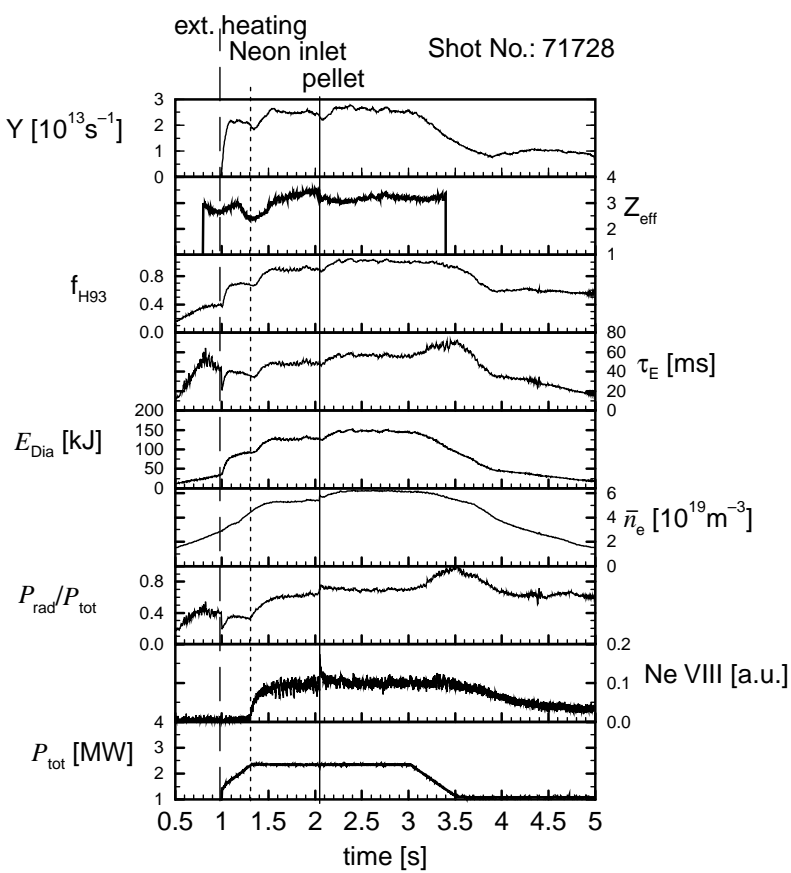

Figure 1. Example of pellet fuelling into an RI mode discharge, showing as a function of time: the total heating power $P_{t o t}$, brilliance of the Ne VIII line, radiated power fraction $\gamma$, line averaged density $\bar{n}_{e}$, diamagnetic energy $E_{\text {dia }}$, energy confinement time $\tau_{E}, f_{H 93}$, neutron yield $Y$ and $Z_{\text {eff }}$.

shows a flat electron density profile while RI mode is characterized by a peaked electron density profile. In contrast to $\mathrm{H}$ mode, where in most cases a degradation of confinement is seen at high densities, RI mode shows a linear density dependence of energy confinement time leading to the best confinement at the highest densities.

\section{Pellet fuelling in radiative improved discharges}

Experiments in TEXTOR-94 have shown that RI mode plasmas are a favourable target for pellet injection; to our knowledge it is the only strongly heated plasma mode where pellet injection does not disturb the confinement properties but leads to a long lasting, quasi-stationary improved confinement state.

An example of the permanent effect of triggered confinement enhancement by pellet injection is shown in Fig. 1. The discharge is performed at a plasma current of $420 \mathrm{kA}$ and a toroidal magnetic field of $2.23 \mathrm{~T}$. The plasma is heated with ICRH (1.23 MW) and neutral beam (co-direction, 


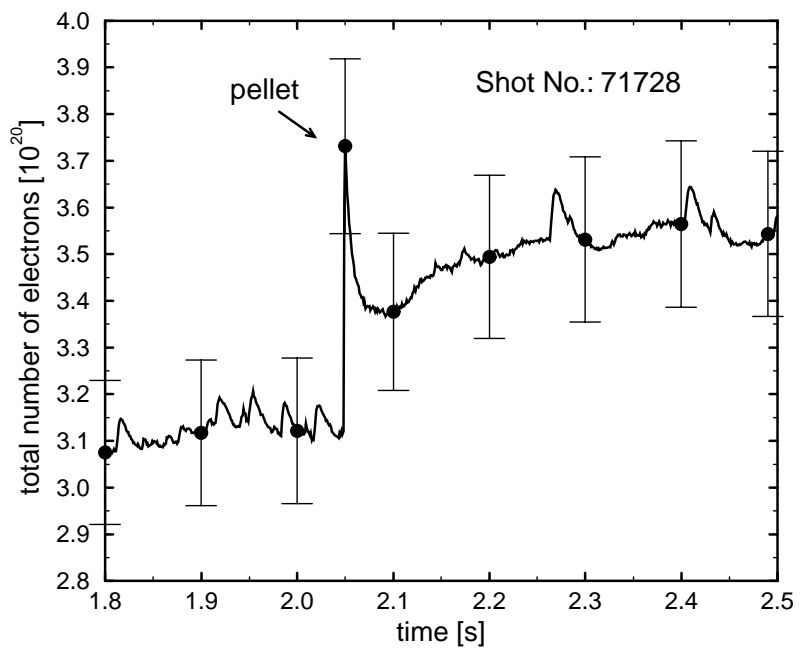

Figure 2. Total number of electrons before and after pellet injection. The pellet causes a sharp increase, a fast decay follows and then a second increase follows.

1.1 MW). Before neon injection, the discharge exhibits L mode confinement. Shortly after the start of neon seeding $(t=1.3 \mathrm{~s})$, the discharge performs a transition to RI mode confinement. The line averaged density and the plasma energy content rise to a stationary value.

A pellet is injected at $t=2.05 \mathrm{~s}$ (velocity $v=$ $960 \mathrm{~m} / \mathrm{s}$, penetration depth $d=27 \mathrm{~cm}$ and particle content $N=7 \times 10^{19}$ deuterium atoms). As shown in Fig. 2, pellet injection results in a strong increase in the total number of electrons. After the initial increase to $3.7 \times 10^{20}$, the number of electrons decreases to about $3.4 \times 10^{20}$ with a time constant of $22 \mathrm{~ms}$. Then the number of electrons starts to increase again and after $300 \mathrm{~ms}$ a new equilibrium state is reached where the total number of electrons stays constant at about $3.5 \times 10^{20}$. An initial decrease in particle content is also observed when a pellet is injected into an L mode discharge; however the slow increase $(150 \mathrm{~ms})$ of the density after the initial decay is a special feature of RI mode discharge conditions and has not yet been observed in any other discharge type.

As can be seen in Fig. 1 the radiated power fraction increases further with pellet injection and relaxes to a higher value than that before the pellet. The line averaged density behaves as the total number of electrons. Together with the density, the plasma energy and confinement time (calculated as $\tau_{E}=E_{d i a} / P_{t o t}$ with $E_{d i a}$ being the energy measured with a compensated diamagnetic loop and $P_{\text {tot }}$ the total heating power including the ohmic heating power) reaches a new quasi-stationary value. The $f_{H 93}$ factor (this factor is the ratio between the measured energy confinement time and the confinement time calculated using the ITER93H scaling) amounts to 0.7 before neon seeding, to 0.85 after neon seeding and to 0.95 in the quasi-stationary phase after pellet injection and has enabled the discharge to reach a confinement quality which is very close to that of ELM-free $\mathrm{H}$ mode. The first quantity shown in Fig. 1 is the neutron rate, which shows an initial decrease shortly after pellet injection but then recovers to a value slightly higher than before.

The concentrations of neon and carbon amount to about $0.9 \%$ in the centre and also remain at this value after the pellet has been injected. A decrease in $Z_{\text {eff }}$ by $18 \%$ is observed, indicating that the concentration of other impurities such as oxygen is reduced. At $t=3 \mathrm{~s}$ the auxiliary heating is reduced and the quasi-stationary high confinement phase ends.

The radiation at the plasma boundary increases after pellet injection as found by bolometric measurements, due to a density increase in the outer third of the plasma radius, even though the neon concentration is constant there. At the plasma edge the temperature and density at the LCFS deduced from the thermal helium beam change only slightly. The recycling hydrogen flux at the toroidal pump limiter surface (ALT-II) increases by $16 \%$; this is the same increase as that of the total number of electrons such that the global particle confinement time stays nearly constant.

In the plasma core, the electron temperature is reduced for a short time interval after injection of the pellet, as shown in Fig. 3. The ion temperature is not influenced at all by the pellet injection although the time resolution of $50 \mathrm{~ms}$ can hide a transient decrease.

The observed improvement of the PEP mode [5$7]$ is sometimes attributed to a rearrangement of the plasma current. Therefore the current profile (and the accompanying safety factor profile) has been measured on TEXTOR-94 by polarimetry. The value of $q$ on-axis $\left(q_{0}\right)$ is also shown in Fig. 3. In the example given, the current distribution (and with this the $q$ profile) remains unchanged after pellet injection.

\subsection{Profile effects}

An important parameter for the interpretation of the confinement increase is the shape of the electron density profile. In Fig. 4 the temporal development of the electron density profile is plotted. The 


\section{J. Hobirk et al.}

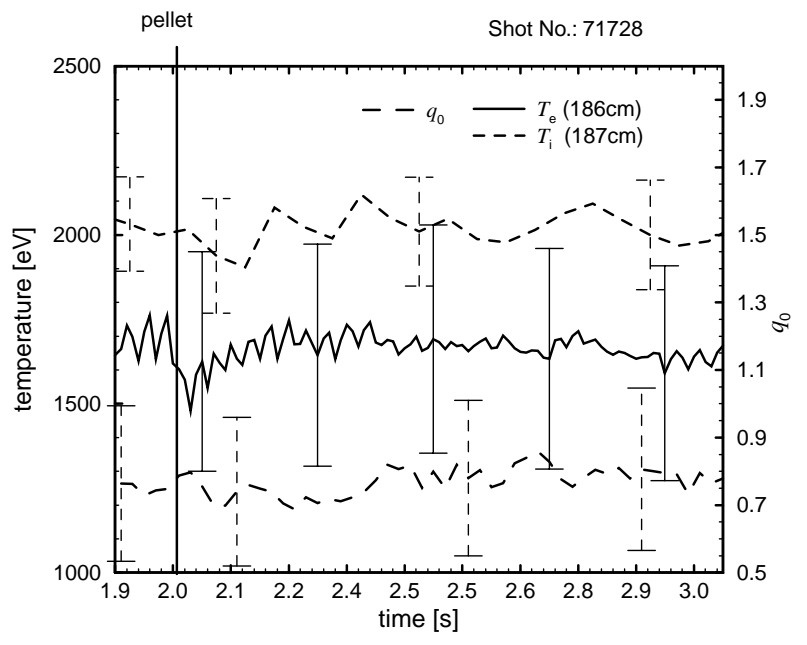

Figure 3. Development of electron and ion temperatures and $q_{0}$ in discharge 71728 .

profiles are derived from Abel inversion and the absolute error is about $10 \%$ in the central part of the profile. Also included in Fig. 4 is the peaking factor, which is defined as $p=n_{e}(0) /\left\langle n_{e}\right\rangle$, where $\left\langle n_{e}\right\rangle$ is volume averaged electron density and $n(0)$ central electron density. The full curve in the lower part of the figure shows the density profile just before pellet injection. This profile is peaked compared with those of high density L mode discharges. The profile at $t=2.05 \mathrm{~s}$ (short dashed curve) is taken near the time when the total number of electrons reaches its maximum. Due to the fact that the pellet is ablated in the outer region of the plasma this profile is broadened. The profile at $t=2.07 \mathrm{~s}$ (long dashed curve) represents the density at the time of the minimum in the total number of electrons. It indicates that most of the particles in the wings of the profile have been transported out of the plasma. Some of the particles have also been transported to the plasma centre as indicated by the beginning of the peaking. The last profile in this plot $(t=2.11 \mathrm{~s}$, dotted line) is taken in the phase where the total number of electrons increases. It shows that particles from the wall have been transported into the bulk plasma. It is still less peaked than the profile before pellet injection. The electron density profiles shown in the upper part of Fig. 4 describe the further peaking of the density profile. After some hundred milliseconds the electron density profile reaches a quasi-stationary shape again. The profile wings do not change in this phase, and a density accumulation is seen inside the $q=3 / 2$ surface. The profiles are more peaked during

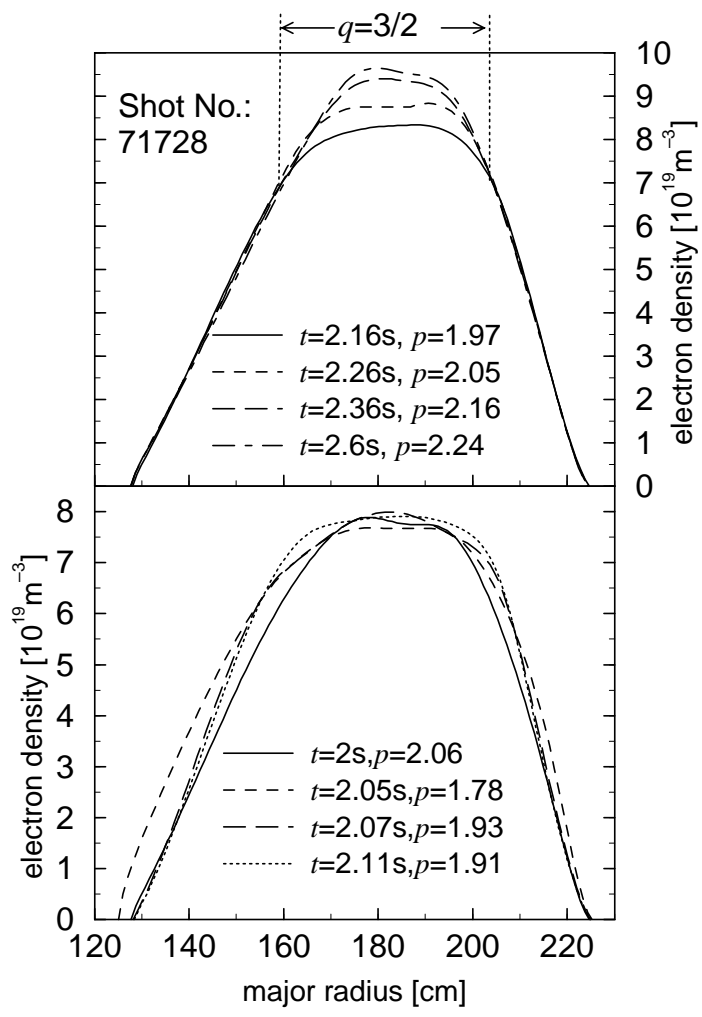

Figure 4. Temporal evolution of the density profile after a pellet has been injected. In the lower part the development of the electron density between 2 and $2.11 \mathrm{~s}$ is shown and in the upper part the development between 2.16 and $2.6 \mathrm{~s}$ is shown.

this phase than in the RI mode phase before pellet injection.

\section{Reaching high $\beta$ with pellets}

Injecting pellets can lead to high confinement and high $\beta$ in TEXTOR-94. In the discharge plotted in Fig. 5, a pellet is injected at $t=1.6 \mathrm{~s}$ and causes an increase in density and energy confinement time. In this discharge, the density does not stay constant after the pellet but increases further; the increase in energy saturates after $1 \mathrm{~s}$ where a normalized beta $\beta_{N}=2$ is reached. The definition of normalized beta is as follows:

$$
\begin{aligned}
& \beta_{N}=\beta_{t} / \frac{I_{p}}{a B_{t}} \\
& \beta_{t}=2 \mu_{0} \frac{\langle p\rangle}{B_{t}^{2}}
\end{aligned}
$$

with $\beta_{t}$ as a percentage, $I_{p}$ in megamps, $a$ in metres and $B_{t}$ in teslas. In this discharge the temperature 


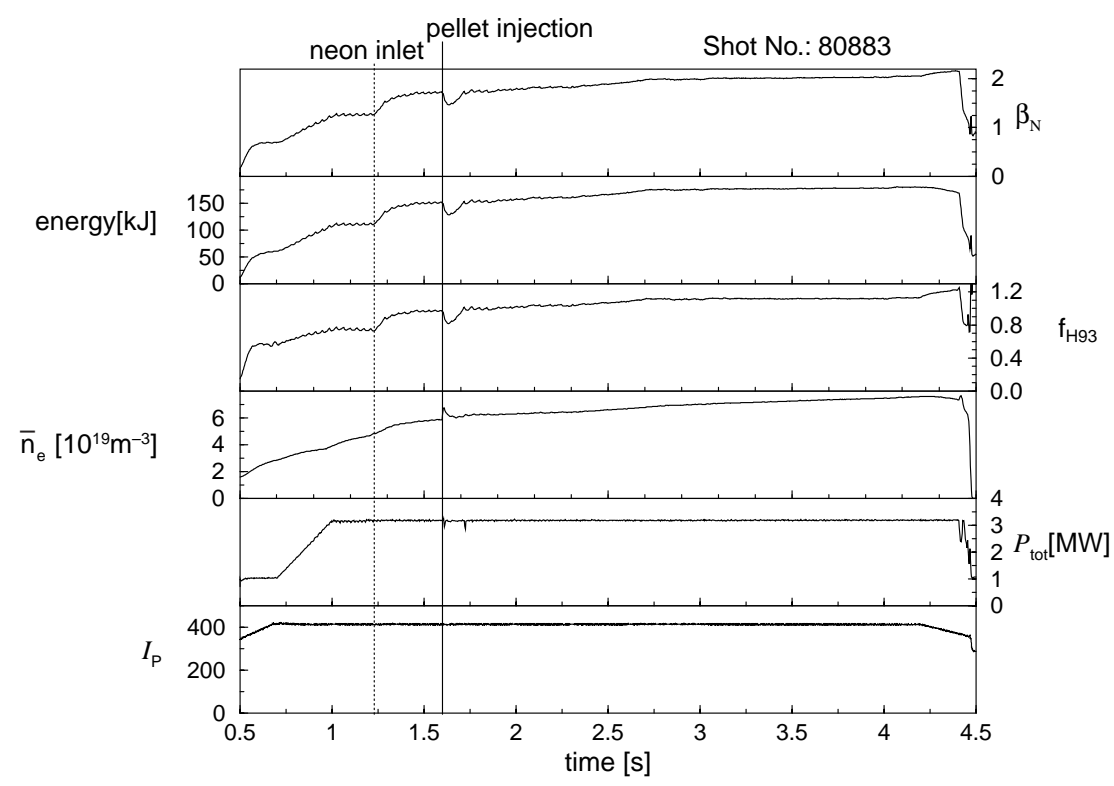

Figure 5. Example of an RI mode discharge with a long and stationary high confinement phase (saturated at high $\beta$ ) triggered by a pellet.

in the region of the $q=3 / 2$ surface is about $1.3 \mathrm{keV}$ so that the resulting $\rho_{p, i}^{*}=0.089$ (defined as $\rho_{p, i}^{*}=$ $\left.\rho_{p, i} / a\right)$ is large and the $\beta$ limit extrapolated from a scaling provided by Ref. [23] is probably not reached. The density increases further but the energy does not increase any longer. For the whole duration of the discharge, strong MARFEs and modes are active which may limit the energy reached, but in contrast to other discharges with MARFEs and mode activity this discharge is stable and the mode activity does not lead to a sudden confinement degradation.

\section{Discussion}

The injection of pellets into RI mode discharges yields no more special features from the pellet ablation point of view. The penetration depth is not different (in the error bars) from the one in discharges without radiation cooling. Multipellet injection experiments (up to three pellets) with a time difference of $500 \mathrm{~ms}$ between the pellets have shown that each injected pellet causes similar effects; they will be treated as single pellets in the following paragraphs.

The influence of pellet injection on the plasma performance is dependent on the neutral pressure near the wall as shown in Fig. 6. With increasing neutral pressure the difference between the energy before pellet injection and after decreases and becomes

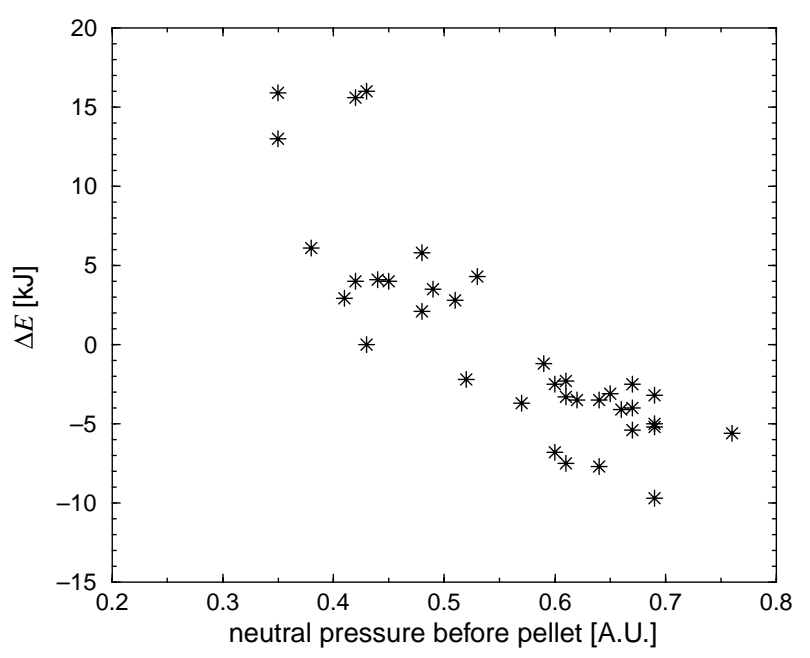

Figure 6. Difference in energy before and $200 \mathrm{~ms}$ after pellet injection as a function of the neutral pressure at the wall.

negative, which means that the energy confinement time falls if the neutral pressure becomes too large. In the multipellet injection experiments the pellets caused an increase in the neutral pressure so that the improvement in energy decreased with each injected pellet.

To study the influence of pellet injection on plasma performance the enhancement factor $f_{H 93}$ is plotted against the Greenwald factor in Fig. 7. The 


\section{J. Hobirk et al.}

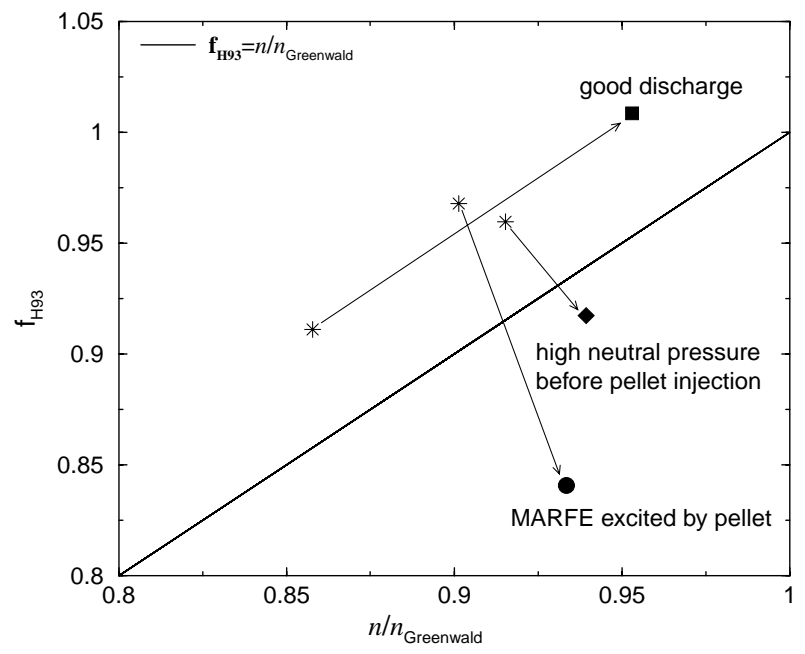

Figure 7. Development of discharges after pellet injection in relation to the RI mode scaling. A discharge with low neutral pressure moving along the scaling (star to closed square), a discharge with high neutral pressure moving across the scaling (star to closed diamond) and a discharge with a MARFE after pellet injection (star to closed circle).

stars represent the discharges before pellet injection and lie a little above the RI mode scaling. The arrows show in which direction the discharge has developed $200 \mathrm{~ms}$ after pellet injection. In a good case, with low neutral pressure, as discussed before, the point representing the discharge is moved parallel to the scaling (closed square). In the case of high neutral pressure the point is moved to a slightly higher density but reduced energy confinement (closed diamond). Another case occurs if a MARFE is excited. The energy is much more reduced (closed circle) than in the other cases.

One reason for the higher energy confinement time is connected to the shape of the density profile. In the discharge with enhanced confinement discussed above, the improvement is connected with a peaking of the density profile. In a comparable discharge with a decrease in the energy confinement time, the density profile does not peak after the pellet is injected. There is a general connection between density profile peaking and improved confinement, shown in Fig. 8. All available discharges are plotted in Fig. 8; the plotted line is a linear fit to the points. On the $x$ axis the difference in peaking factor before and $200 \mathrm{~ms}$ after pellet injection is plotted; values greater than zero represent discharges where a density peaking takes place and values below zero represent discharges with broadened density profiles. The difference in energy

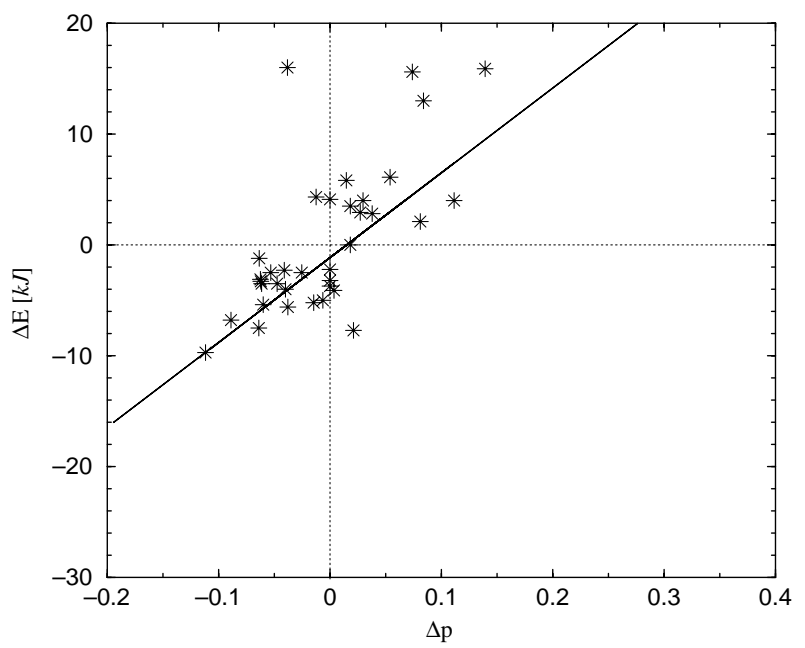

Figure 8. Difference in energy before and $200 \mathrm{~ms}$ after pellet injection as a function of the difference in peaking factor before and $200 \mathrm{~ms}$ after pellet injection.

content between $200 \mathrm{~ms}$ after injection of the pellet and the initial energy content is shown on the $y$ axis.

Apart from the negative effect of higher neutral pressure in the midplane and the necessity of more peaked density profiles, no other relationship of the difference in energy before and after the injected pellet could be found. Thus about 20 parameters (such as the electron density at the scrape-off layer, total radiation power, heating power, penetration depth of the pellet, size of the pellet, position of the plasma) were investigated.

As a mechanism for the improvement during RI mode in TEXTOR-94 suppression of the toroidal ion temperature gradient (ITG) turbulence mode $[24,25]$ has been proposed. Basic ingredients for the development or stabilization of the ITG mode [26] are the density and ion temperature gradient and the concentration of impurity species $Z_{i}$ in the ionization state $i$.

Within this picture of ITG stabilization and confinement, the pellet acts primarily on the gradients of density and temperature: during the ablation process, the energy of the plasma is used to ionize and heat the ablated gas of the pellet. Therefore, locally density is increased and temperature is reduced. On a longer timescale, particles are deposited inside the good confinement zone and furthermore particles are lost from the outer part of the gradient zone so that the profile is locally steepened. The corresponding stability parameter for the ITG mode $\eta_{i}$ (defined here as $\left.\eta_{i}=\nabla_{r}\left(\ln T_{i}\right) / \nabla_{r}\left(\ln n_{e}\right)\right)$ is reduced to below 
1 in the core. The ion temperature is constant in time and the density gradient is increased; this extends the zone with low $\eta_{i}$ to the centre of the plasma. We assume that the local modification of the gradient can be a trigger for a local further suppression of the toroidal ITG mode; if this stabilization has taken place, the confinement of the plasma is further increased and particles can accumulate in the core. This process would then explain the relatively slow peaking of the density profile after pellet injection. Once good confinement is reached, the favourable conditions remain stable and are maintained to the end of the TEXTOR-94 discharge.

\section{Conclusions}

The experiments described have shown that the injection of deuterium pellets in RI mode discharges leads to an increase of plasma confinement times if no MARFEs are excited and the neutral pressure is low. The most likely explanation is that the injected pellet does not create a new plasma mode of improved confinement but that it continues RI mode to the upper limit. The confinement improvement is held quasi-stationary up to the end of the RI mode plasma phase.

\section{References}

[1] Milora, S.L., Houlberg, W.A., Lengyel, L.L., Mertens, V., Nucl. Fusion 35 (1995) 657.

[2] Greenwald, M., et al., Phys. Rev. Lett. 53 (1984) 352.

[3] Kaufmann, M., et al., Nucl. Fusion 28 (1988) 827.

[4] Milora, S.L., et al., in Controlled Fusion and Plasma Physics (Proc. 16th Eur. Conf. Venice, 1989), Vol. 13B, Part I, European Physical Society, Geneva (1989) 91.

[5] Smeulders, P., et al., Nucl. Fusion 35 (1995) 225.

[6] Baylor, L.R., et al., Nucl. Fusion 37 (1997) 127.

[7] Maget, P., Garbet, X., Géraud, A., Joffrin, E., in Controlled Fusion and Plasma Physics (Proc. 24th Eur. Conf. Berchtesgaden, 1997), Vol. 21A, Part I, European Physical Society, Geneva (1997) 173.

[8] Gruber, O., et al., Plasma Phys. Control. Fusion 30 (1988) 1611.
[9] Lang, P.T., et al., Phys. Rev. Lett. 79 (1997) 1487.

[10] Kupschus, P., et al., in Controlled Fusion and Plasma Physics (Proc. 24th Eur. Conf. Berchtesgaden, 1997), Vol. 21A, Part I, European Physical Society, Geneva (1997) 45.

[11] Kapralov, V.G., Rozhansky, V.A., Khlopenkov, K.V., in Controlled Fusion and Plasma Physics (Proc. 22nd Eur. Conf. Bournemouth, 1995), Vol. 19C, Part I, European Physical Society, Geneva (1995) 117.

[12] Askinazi, L.G., et al., Phys. Fluids B 5 (1993) 2420.

[13] Corbett, W.J., et al., J. Nucl. Mater. 162-164 (1989) 660.

[14] Dippel, K.H., et al., in Plasma Physics and Controlled Nuclear Fusion Research 1988 (Proc. 12th Int. Conf. Nice, 1988), Vol. 1, IAEA, Vienna (1989) 453.

[15] Goebel, G.M., et al., J. Nucl. Mater. 162-164 (1989) 115.

[16] Samm, U., et al., in Controlled Fusion and Plasma Physics (Proc. 18th Eur. Conf. Berlin, 1991), Vol. 15C, Part III, European Physical Society, Geneva (1991) 137.

[17] Koslowski, H.R., Soltwisch, H., Fusion Eng. Des. 34\&35 (1997) 143.

[18] Krämer-Flecken, A., Waidmann, G., de Vries, P.C., in Electron Cyclotron Emission and Electron Cyclotron Resonance Heating (Proc. 10th Joint Workshop Ameland, 1997).

[19] Kohlhaas, W., et al., Fusion Technol. 17 (1990) 762.

[20] Disdier, F., et al., Fusion Technol. 19 (1992) 467.

[21] Messiaen, A.M., et al., Phys. Rev. Lett. 77 (1996) 2487.

[22] Messiaen, A.M., et al., Phys. Plasmas 4 (1997) 1690.

[23] Koslowski, H.R., et al., Nucl. Fusion 40 (2000) 821.

[24] Tokar', M.Z., Jaspers, R., Unterberg, B., Nucl. Fusion 38 (1998) 961.

[25] Tokar', M.Z., et al., Plasma Phys. Control. Fusion 41 (1999) L9.

[26] Hassam, A.B., Antonsen, T.M., Drake, J.F., Guzdar, P.N., Phys. Fluids B 2 (1990) 1822.

(Manuscript received 13 August 1999

Final manuscript accepted 7 April 2000)

E-mail address of J. Hobirk:

joerg.Hobirk@ipp-garching.mpg.de

Subject classification: F2, Te; J2, Te 